\title{
Research on Fatigue Damage of Compressor Blade Steel KMN-I Using Nonlinear Ultrasonic Testing
}

\author{
Pengfei Wang, ${ }^{1,2,3}$ Weiqiang Wang, ${ }^{1,2,3}$ and Jianfeng $\mathrm{Li}^{1,4}$ \\ ${ }^{1}$ School of Mechanical Engineering, Shandong University, 17923 Jingshi Road, Jinan, China \\ ${ }^{2}$ Engineering and Technology Research Center for Special Equipment Safety of Shandong Province, 17923 Jingshi Road, Jinan, China \\ ${ }^{3}$ Research Center of Safety Guarantee and Assessment to Special Equipment, Shandong University, 17923 Jingshi Road, Jinan, China \\ ${ }^{4}$ Key Laboratory of High Efficiency and Clean Mechanical Manufacture of Ministry of Education, 17923 Jingshi Road, Jinan, China
}

Correspondence should be addressed to Weiqiang Wang; wqwang@sdu.edu.cn

Received 21 June 2017; Revised 13 September 2017; Accepted 26 September 2017; Published 19 October 2017

Academic Editor: M. I. Herreros

Copyright (c) 2017 Pengfei Wang et al. This is an open access article distributed under the Creative Commons Attribution License, which permits unrestricted use, distribution, and reproduction in any medium, provided the original work is properly cited.

\begin{abstract}
The fatigue damage of compressor blade steel KMN-I was investigated using nonlinear ultrasonic testing and the relation curve between the material nonlinearity parameter $\beta$ and the fatigue life was obtained. The results showed that the nonlinearity parameter increased first and then decreased with the increase of the fatigue cycles. The microstructures were observed by scanning electron microscopy (SEM). It was found that some small defects like holes and pits appeared in the material matrix with the increase of the fatigue cycles, and the nonlinearity parameter increased correspondingly. The nonlinearity parameter reached the peak value when the microcracks initiated, and the nonlinearity parameter began to decrease when the microcracks further propagated to macrocracks. Therefore, it is proved that the nonlinearity parameter can be used to characterize the initiation of microcracks at the early stage of fatigue, and a method of evaluating the fatigue life of materials by nonlinear ultrasonic testing is proposed.
\end{abstract}

\section{Introduction}

The centrifugal compressor impeller is a typical high-speed rotating mechanical component, which must have high requirements for the performance of the materials due to the poor service conditions. In recent years, major failure accidents of centrifugal compressor impeller occurred, so the damage detection and life assessment of the impellers become more and more important. It is shown that the damage and performance degradation of the materials at the early stage occupy $80 \%-90 \%$ of the whole fatigue life. The common nondestructive testing can only detect the macroscopic defects (cracks, inclusions, stomata, etc.), while the sensitivity to the early damage of the materials and structures is relatively low, and it cannot detect the small-scale damage such as microdefects and microcracks, especially the hidden damage in the interior of the materials. The recent research shows that the degradation of the material properties at the early stage is always accompanied by some form of nonlinear mechanical behavior, which causes the generation of the nonlinear harmonic waves [1-4]. Therefore, the nonlinear ultrasonic testing overcomes the shortage of the common nondestructive testing, which can effectively characterize the microdefect and hidden damage [5, 6]. In 1999, Jhang and Kim [7] researched the relationship between the mechanical properties degradation of the material and the nonlinearity parameter under tensile load. In 2010, Kawashima et al. [8] detected the nonmetallic inclusions in the continuous casting steels using a nonlinear scanning system. In 2012, Cremer et al. [9] carried out the in situ observation of the very high cycle fatigue damage evolution of aluminum alloy welded joints using nonlinear ultrasonic technology. In 2015, Li et al. [10] carried out the in situ observation of the very high cycle fatigue properties of aluminum alloy and the results show that the ultrasonic nonlinearity parameter is more sensitive to the fatigue damage (such as the initiation and propagation of the cracks). In addition, Solodov et al. pointed out that contact acoustic nonlinearity (CAN) mechanism of cracked faces generates far higher harmonics. Nonclassical effects are due to substantially asymmetric stiffness characteristics of the interface for normal stress that results in specific CAN. The asymmetry in the contact restoring forces causes the stiffness 
parametric modulation and instability of oscillations, which results in the generation of subharmonic acoustic wave. Nonlinear reflection technique provides primary detection of the invisible in linear NDE fractured defects, which are the sources of CAN and the forerunners of the main cracking and subsequent fracture $[11,12]$.

Currently the fatigue test of the compressor blades mainly includes the resonance fatigue test and the ultrasonic fatigue test, in which the ultrasonic fatigue test also uses the ultrasonic transducer to resonate the specimen. However, not all the fatigue failure of the blades is caused by resonance in the actual service condition. The form of the load is not single because the specimens may be subject to bending, tensile, torsion, and their composite form load, so the current vibration fatigue test cannot simulate the actual service condition of the blades. In 2006, Q. Yao and J. Yao [13] divided the structural fatigue into two kinds of problems: static fatigue and vibration fatigue. When the frequency distribution of the dynamic alternating load (e.g., vibration, impact, and noise load) is similar to the natural frequency distribution of the structure, resonance of the structure leads to fatigue failure, which is called vibration fatigue, while the static fatigue is fatigue failure caused by the forced vibration of the structure under the alternating load, in which time the structure did not resonate. Most of the blades' fatigue test is carried out using vibration testing or ultrasonic fatigue testing, while the static fatigue test of the compressor blades is relatively less. In 2006, Rajasekaran and Nowell [14] carried out the multiaxis fatigue test (tensile and bending vibration load) on the root of the blades, which simulated the multiaxis fatigue load of the compressor blades under the actual service condition. In 2012, Liu and He [15] designed the force excitation vibration fatigue test system using the exciter directly acting on the specimen and proposed a test method of the vibration fatigue life.

\section{Theoretical Basis of Nonlinear Ultrasonic Testing}

There is a nonlinear term in the wave equation of the sound waves propagating in the medium. The degradation of the material properties at the early stage is always accompanied by some form of nonlinear mechanical behavior, which causes the generation of the nonlinear harmonic waves. These higher harmonics waves are very sensitive to the early damage of the materials and structures.

Due to the nonlinearity of the solid medium, the stressstrain relationship exhibits a nonlinear characteristic, which can be expressed as

$$
\sigma=E \varepsilon\left(1-\frac{\beta \varepsilon}{2}+\frac{\delta \varepsilon^{2}}{3}\right),
$$

where $\sigma$ is the stress, $\varepsilon$ is the strain, $E$ is Young's modulus, and $\beta$ and $\delta$ are the parameters describing the nonlinearity of the material, which are the second-order nonlinearity parameter and the third-order nonlinearity parameter [16-18]. In order to describe the generation of the second harmonic or higher harmonics in the solid medium, it is assumed that a single frequency sinusoidal ultrasonic signal propagates in the material. Due to the nonlinearity of the solid medium, the nonlinear interaction between the ultrasonic waves and the solid medium causes the generation of the high harmonics. In this case, the one-dimensional nonlinear wave equation in the solid medium can be expressed as (the first two items of the series expansion)

$$
\begin{aligned}
\rho \frac{\partial^{2} u}{\partial t^{2}} & =\frac{\partial \sigma}{\partial x} \\
\frac{\partial^{2} u}{\partial t^{2}} & =c^{2}\left(1-\beta \frac{\partial u}{\partial x}\right) \frac{\partial^{2} u}{\partial x^{2}},
\end{aligned}
$$

where $c=\sqrt{E / \rho}$ is the velocity of the longitudinal wave, $\rho$ is the density of the medium, $x$ is the propagation distance of the longitudinal wave, and $u$ is the displacement of the particle at $x$. The solution of (3) $u=u_{0}+u_{1}$ can be calculated using the second-order perturbation method $\left(u_{0}\right.$ is the solution when $\beta=0$ and $u_{1}$ is the first-order perturbation solution). Through the iterative process, we can find the concrete expression of (3)

$$
\begin{aligned}
u(x, t)= & A_{1} \sin (k x-\omega t) \\
& +\frac{A_{1}^{2} k^{2} \beta x}{8} \cos 2(k x-\omega t),
\end{aligned}
$$

where $A_{1}$ is the amplitude of the fundamental frequency signal, $k$ is the wave number $(k=2 \pi / \lambda, \lambda$ is the wavelength), and $\omega$ is the angular frequency. So the amplitude of the second harmonic propagating in the material, $A_{2}$, is $A_{1}{ }^{2} k^{2} \beta x / 8$.

Therefore, the ultrasonic nonlinearity parameter can be obtained by measuring the ultrasonic fundamental frequency signal and the high harmonic signal. The second-order nonlinear parameter $\beta$ can be expressed as

$$
\begin{aligned}
& \beta=\frac{8}{k^{2} x} \frac{A_{2}}{A_{1}^{2}}, \\
& \beta \propto \frac{A_{2}}{A_{1}^{2}} .
\end{aligned}
$$

\section{Experimental Material and Method}

3.1. Experimental Material. KMN-I is an important material for the manufacture of centrifugal compressor impellers due to good corrosion resistance, high strength, and good weld ability. It is a low alloy steel. The experiment material is supplied by Shenyang Blower Group, and KMN-I is the internal code of this material. The chemical composition of KMN-I is similar to $15 \mathrm{Cr} 2 \mathrm{Mol}$. The main chemical compositions and mechanical properties KMN-I are shown in Tables 1 and 2.

The heat treatment process is shown in Table 3. The microstructure of KMN-I is mainly acicular and lath tempered troostite, as shown in Figure 1.

3.2. Preparation of Fatigue Specimens. The shape of the fatigue specimen is shown in Figure 2, and the thickness of the specimen is $2 \mathrm{~mm}$. The surface of the specimen was 
TABLE 1: Mechanical properties of KMN-I.

\begin{tabular}{lcccc}
\hline$R_{m}(\mathrm{MPa})$ & $R_{p 0.2}(\mathrm{MPa})$ & $E(\mathrm{GPa})$ & $\rho\left(\mathrm{kg} / \mathrm{m}^{3}\right)$ & $\begin{array}{c}H V \\
\left(\mathrm{kgf} \cdot \mathrm{mm}^{-2}\right)\end{array}$ \\
\hline 1193 & 1072 & 205 & 7840 & 335 \\
\hline
\end{tabular}

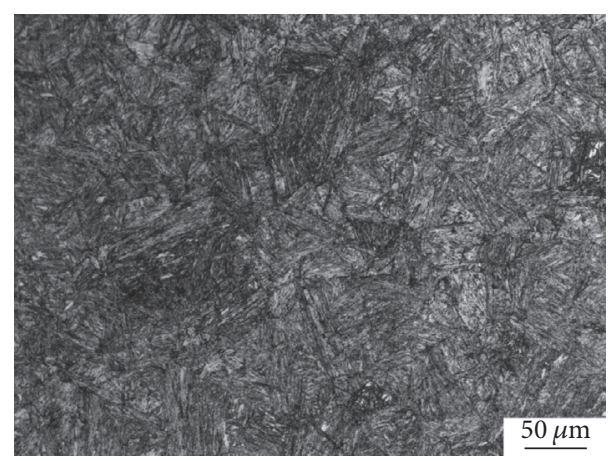

Figure 1: Microstructure of KMN-I.

mechanically polished with emery papers to keep the surface of each specimen consistent before the fatigue test.

In order to simulate the actual service condition of the blades, we modified the ET-10d-240 vibration testing and carried out tensile, bending, torsion, and their composite fatigue test using the designed fixture. This piece of equipment has been awarded a national invention patent (Patent Number ZL 201410271461.2). The two groups of specimens in this experiment were three-point bending fatigue and tensile-bending fatigue, as shown in Figure 3. The operational principle is as follows: the two ends of the three-point bending fatigue specimen were fixed on the fixture of the base, and then periodic sinusoidal load was carried out on the middle of the specimen using the vibration testing and the rigid ball of the fixture. Therefore the specimen was subjected to bending fatigue alternating load. In another group of specimens, the two ends of the tensile-bending fatigue specimen were fixed on the fixture of the base and a horizontal preload was applied to the end of the specimen; then periodic sinusoidal load was carried out on the middle of the specimen using the vibration testing and the rigid ball of the fixture. Therefore the specimen was subjected to tensile-bending fatigue load. Fatigue load of the KMN-I specimen was carried out by vibration testing, and the fatigue specimens with different cycles were obtained. The acceleration control model was used in this experiment. The test temperature is room temperature; the load waveform is sine wave; the load frequency is $40 \mathrm{~Hz}$; the acceleration is $8 \mathrm{~m} / \mathrm{s}^{2}$; the stress of bending test is about $500 \mathrm{MPa}$; and the stress of tensilebending test is about $580 \mathrm{MPa}$.

3.3. Nonlinear Ultrasonic Testing. In this experiment, the longitudinal wave incidence method was used to excite and accept the ultrasonic Lamb wave, which was accurate, effective, and easy to excite. Ultrasonic Lamb wave measurement of KMN-I specimens with different fatigue cycles was carried out using RAM-5000 high energy ultrasonic system, as shown in Figure 4. The pulse signal was excited by the high energy ultrasonic system and went through the attenuator for energy regulation. Then the signal went through the lowpass filter for signal filtering and finally was transmitted to the ultrasonic piezoelectric transducer. The voltage signal was converted to ultrasonic vibration signal and transmitted through the specimen. After the propagation of a certain distance in the specimen, the ultrasonic signal under the influence of the nonlinear stress-strain was received by the piezoelectric transducer with an integer multiple excited frequency. Then the signal was transmitted into the oscilloscope and computer for data display, process, and analysis after the high-pass filter and preamplifier. The launch probe was a narrowband piezoelectric transducer with a center frequency of $2.25 \mathrm{MHz}$, and the receiver probe was a wideband piezoelectric transducer with a center frequency of $5 \mathrm{MHz}$. The second harmonic amplitude is generally far smaller than the fundamental amplitude. In order to better observe the characteristics of the second harmonic, we added a $4 \mathrm{MHz}$ high-pass filter to increase the second harmonic amplitude. The frequency of the excitation signal was $2.2 \mathrm{MHz}$. The incident angle was $27^{\circ}$. Two probes were fixed with a specific device to ensure that the pressure of these two probes surface was controlled at a relatively close level by a pressure sensor.

In order to ensure the consistency of the measurement, the probe and the specimen were thin layer coupling with glycerol. The material nonlinearity of the coupling agent can be neglected because the coupling layer was very thin. Considering that the oblique plexiglass also had the elasticity nonlinear effect, the propagation distance of the excited longitudinal wave in the oblique should be as short as possible. The signals obtained by the experiment included the fundamental and high-order mode Lamb waves, which can be processed by fast Fourier transform (FFT) and short-time Fourier transform (STFT). Therefore, we can calculate the amplitude of the fundamental frequency Lamb and second harmonic Lamb wave [19].

\section{Experimental Results}

4.1. Dispersion Curve. The efficiency of the second harmonic generation was very low due to the dispersion characteristics of the ultrasonic guided wave. The second harmonic signal was very weak and inconvenient to measure. If the phase velocity of the fundamental frequency Lamb wave mode excited in the specimen was equal to the phase velocity of the double-frequency Lamb wave mode, the second harmonic signal was relatively easy to measure. Therefore, the dispersion curves of the fundamental frequency and the second harmonic Lamb wave in the KMN-I specimen were measured, as shown in Figure 5. In order to excite the second harmonic Lamb wave with the accumulation effect, the fundamental Lamb wave with excitation frequency of $2.25 \mathrm{MHz}$ was selected, in which case the fundamental frequency and the second harmonic Lamb wave had the same phase velocity. Besides, some researchers consider that the group velocity also should be matched, so we meet the group velocity matching conditions in the following experiment. 
TABLE 2: Chemical composition of KMN-I (wt.\%).

\begin{tabular}{lcccccc}
\hline $\mathrm{C}$ & $\mathrm{Mn}$ & $\mathrm{Si}$ & $\mathrm{Mo}$ & $\mathrm{Cr}$ & $\mathrm{P}$ & \\
\hline $0.13-0.18$ & $0.5-0.8$ & $0.17-0.37$ & $0.9-1.1$ & $2.2-2.5$ & $\leqslant 0.030$ & $\leqslant 0.030$ \\
\hline
\end{tabular}

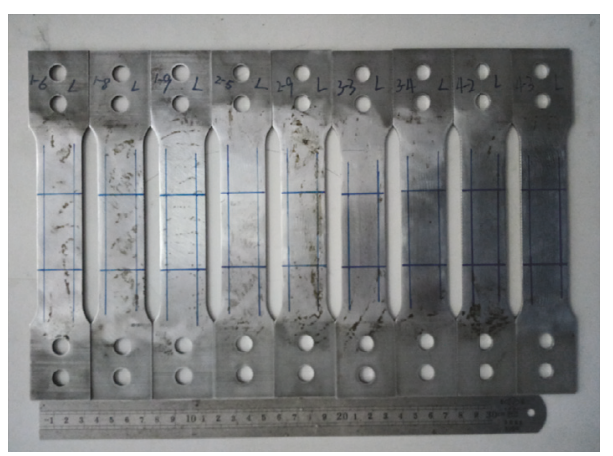

(a) Specimen shape

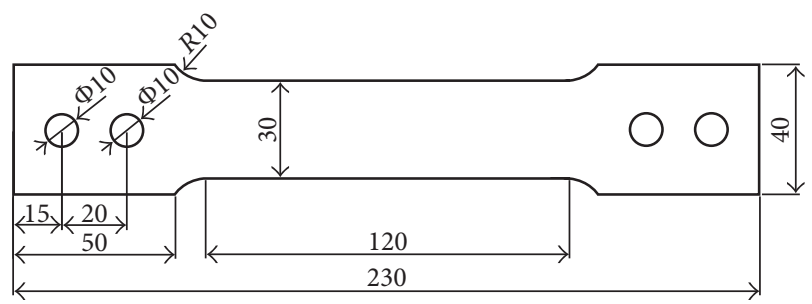

(b) Geometric dimensions

FIGURE 2: Shape and dimensions of KMN-I fatigue specimen.

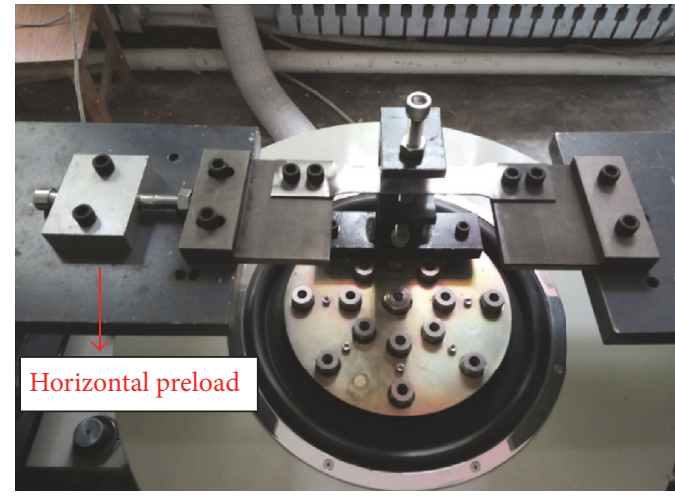

(a) Tensile-bending fatigue test

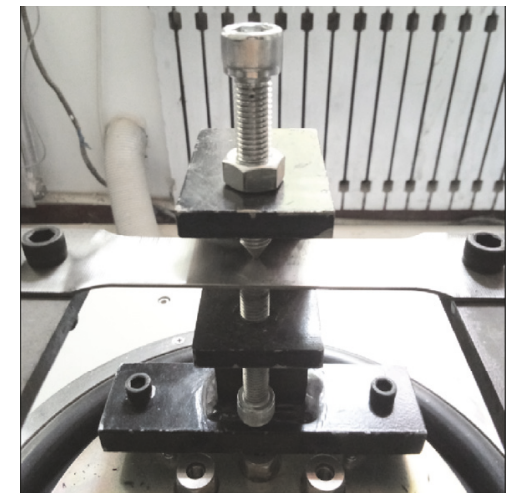

(b) Three-point bending fatigue test

Figure 3: Vibration testing and fixture.

TABLE 3: Heat treatment process.

\begin{tabular}{lccc}
\hline $\begin{array}{l}\text { Heat } \\
\text { treatment }\end{array}$ & $\begin{array}{c}\text { Temperature } \\
\left({ }^{\circ} \mathrm{C}\right)\end{array}$ & $\begin{array}{c}\text { Holding time } \\
(\mathrm{h})\end{array}$ & $\begin{array}{c}\text { Cooling } \\
\text { method }\end{array}$ \\
\hline Quenching & $970 \pm 10$ & $2.5-3$ & Oil cooling \\
Tempering & $570 \pm 10$ & $4-5$ & Air cooling \\
\hline
\end{tabular}

4.2. Signal Validity Verification. Before the nonlinear ultrasonic Lamb wave measurement of the KMN-I specimens with different fatigue damage, it was necessary to measure and verify the amplitude response of the fundamental and doublefrequency Lamb wave of RAM5000 high energy ultrasonic system to ensure that the measured second harmonic signal was caused by the nonlinearity of the tested material, rather than the measurement system.

Nonlinear ultrasonic testing was carried out on KMNI fatigue specimens with different fatigue cycles and the time-domain signals of bending fatigue and tensile-bending fatigue specimens were obtained, as shown in Figure 6.
Then the time-domain signals were processed with STFT, and the STFT time-frequency energy spectrum image of KMN-I specimens was obtained, as shown in Figure 7. The STFT energy spectrum is represented by 256 levels of gray scale and the deeper color means higher amplitude. It can be seen that the received Lamb wave signal is mainly $S_{1}$ mode and its generated second harmonic $S_{2}$ mode. The frequencies are $2.25 \mathrm{MHz}$ and $4.5 \mathrm{MHz}$, respectively. Since the fundamental frequency amplitude of the nonlinear ultrasonic Lamb wave, $A_{1 f}$, and the second harmonic amplitude, $A_{2 f}$, was obtained, the normalized ultrasonic nonlinearity parameter $A_{2 f} / A^{2}{ }_{1 f}$ can be calculated.

There was a linear relationship between $A_{2 f} / A^{2}{ }_{1 f}$ and the propagation distance when the incident voltage remained constant. In order to confirm that the measured second harmonic signal came from the nonlinear Lamb wave signal generated in the specimen, we keep the incident voltage constant and change the distance between the incident transducer and the receive transducer (from $30 \mathrm{~mm}$ to $70 \mathrm{~mm}$ ). From Figure 8 it can be seen that $A_{2 f} / A^{2}{ }_{1 f}$ basically shows 


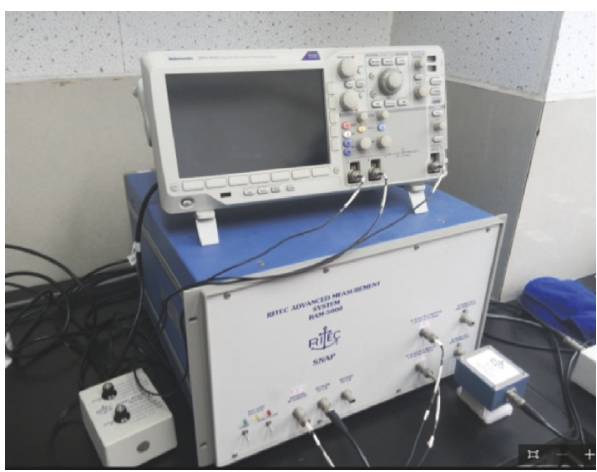

(a) RAM-5000 high energy ultrasonic system

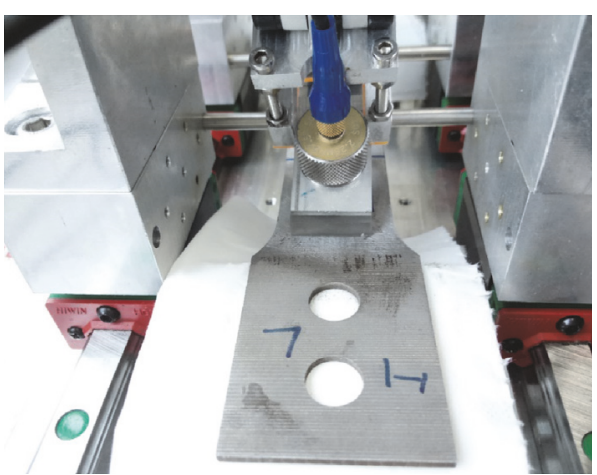

(b) Experiment procedure

FIGURE 4: Nonlinear ultrasonic test system.

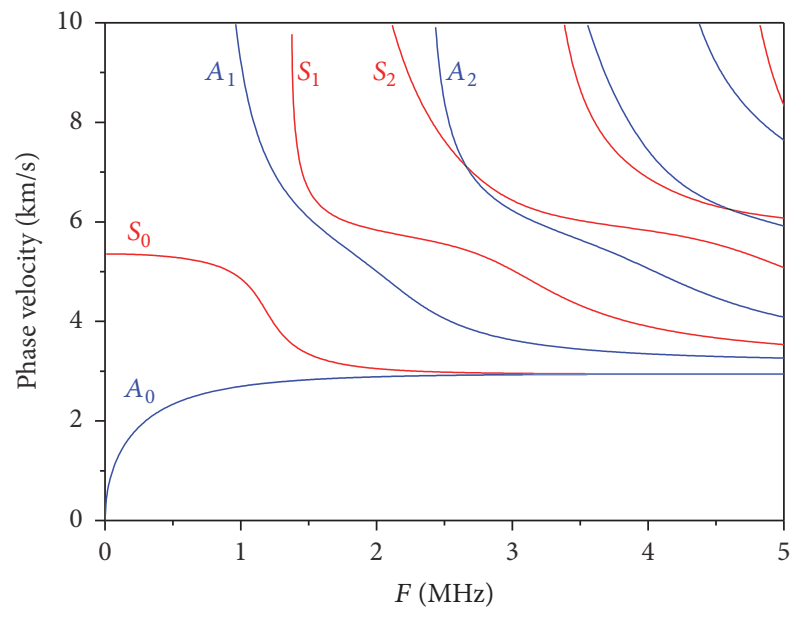

(a) Phase velocity dispersion curve

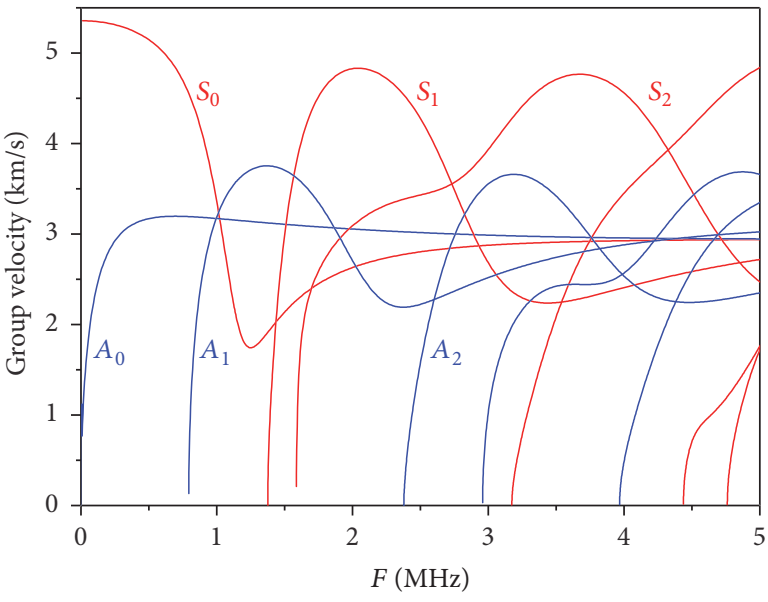

(b) Group velocity dispersion curve

FIgURE 5: Dispersion curve of the Lamb wave in the KMN-I specimen.

a linear increase trend with the increase of the propagation distance. The result shows that the second harmonic signal received by the receive transducer is the second harmonic signal generated by the fundamental frequency Lamb wave propagation in the specimen, rather than the oblique or coupling agent.

4.3. $\beta$-N Curve. $A_{2 f} / A^{2}{ }_{1 f}$ was used as the normalized nonlinear parameter to characterize the nonlinear variation of the material due to fatigue damage, and we can obtain the relationship between the nonlinearity parameter $\beta$ and fatigue cycles, as shown in Figure 9.

For the bending fatigue test, it can be found that the nonlinearity parameter increased first and then decreased with the increase of the fatigue cycles. In the early stage of the fatigue life (before $10^{6}$ cycles), the nonlinearity parameter gradually increased with the increase of the fatigue cycles, and the nonlinearity parameter reached the peak value when the microcracks initiated. In the late stage of the fatigue life (after $10^{6}$ cycles), the nonlinearity parameter began to decrease. The results indicated that there was a correspondence between the nonlinearity parameter and the fatigue damage of the specimen. The fatigue damage of the specimen continuously accumulated with the increase of the fatigue cycles. When the ultrasonic Lamb wave propagated inside the specimen, the second harmonic excited in the damage area increased correspondingly, which led to the increase of the nonlinearity parameter. However, the attenuation coefficient of the material increased due to the cracks, and the nonlinearity parameter decreased correspondingly. This was the reason for the decrease of the nonlinearity parameter in the late stage of the fatigue life. In the late stage of fatigue life, the microholes and microcracks inside the material gradually increase and aggregate, which will result in the increase of sound energy attenuation [20]. In addition, according to Hikata dislocation string model, the dislocation string length increases when the stress is high, and the attenuation of the second harmonic will increase due to the increase of dislocation string length $[21,22]$.

For the tensile-bending fatigue test, the relationship curve also showed the similar trend: the nonlinearity parameter increases first and then decreases with the increase of the fatigue cycles. The difference between the two tests was 


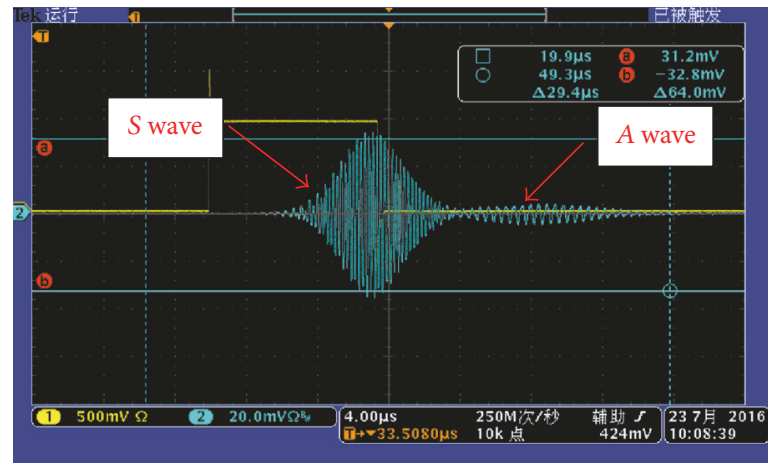

FIgURE 6: Time-domain signals of KMN-I specimen $\left(N_{f}=5 \times 10^{6}\right)$.

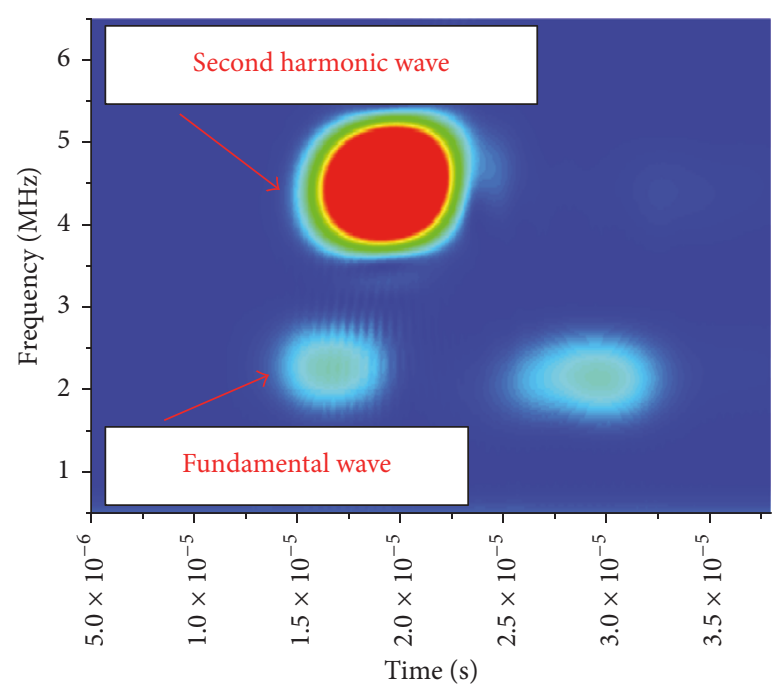

FIGURE 7: STFT spectrograms of KMN-I specimen Lamb wave signals $\left(N_{f}=5 \times 10^{6}\right)$.

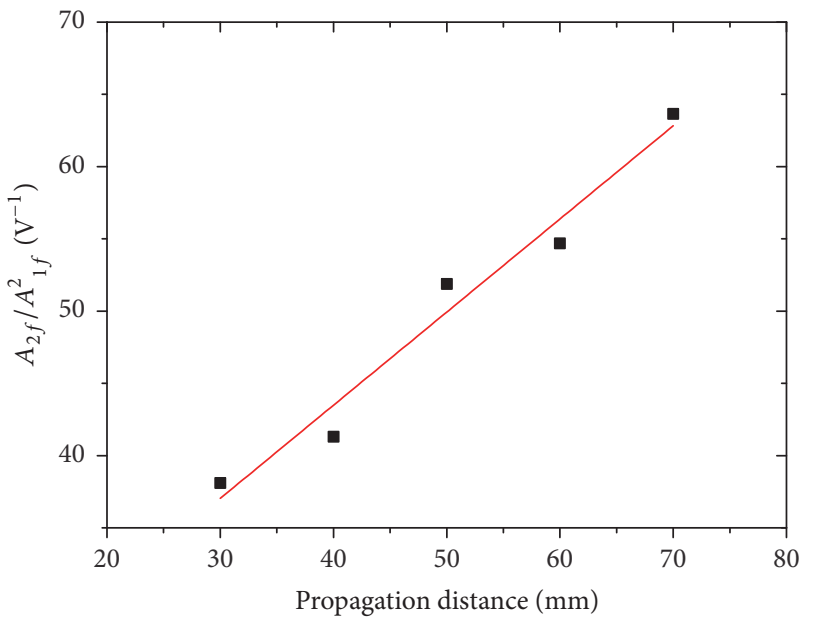

- Experimental data

- Linear fitting curve

FIGURE 8: Relationship between $A_{2 f} / A^{2}{ }_{1 f}$ and the propagation distance.

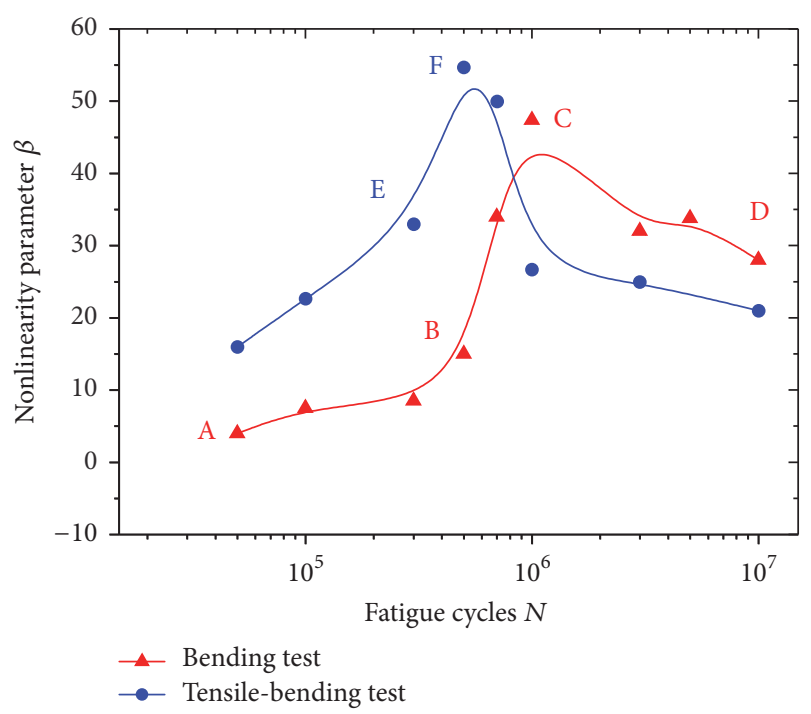

FIGURE 9: Relationship between nonlinearity parameter and fatigue cycles.

mainly the amplitude of the nonlinearity parameter. The load in the dangerous location of the tensile-bending specimen was larger due to the tensile load, and the numbers of the fatigue defects generated in this location were more than the bending specimen. Therefore, the amplitude of the nonlinearity parameter was relatively high.

Compared with the two tests, it can be found that the peak value of the nonlinearity parameter measured by the tensile-bending fatigue test was larger than that of the bending fatigue test. In the early stage of the fatigue life, the nonlinearity parameter measured by the tensile-bending fatigue test was generally higher than that of the bending fatigue test under the same fatigue cycle. This was because the stress in the dangerous location of the specimen increased due to the lateral tensile load. The excited second harmonic was more obvious under the same fatigue cycle, resulting in an increase of the nonlinearity parameter. In addition, the $\beta$ $N$ curve measured by the tensile-bending fatigue test shifted leftward relative to the $\beta-N$ curve measured by the bending fatigue test. The stress in the dangerous location of the 


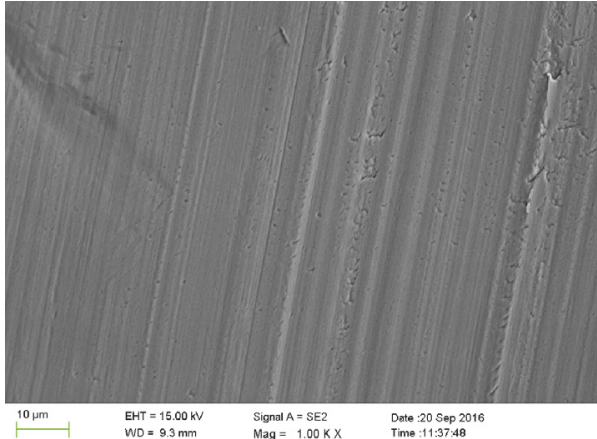

(a) Microstructure of point A in Figure 9

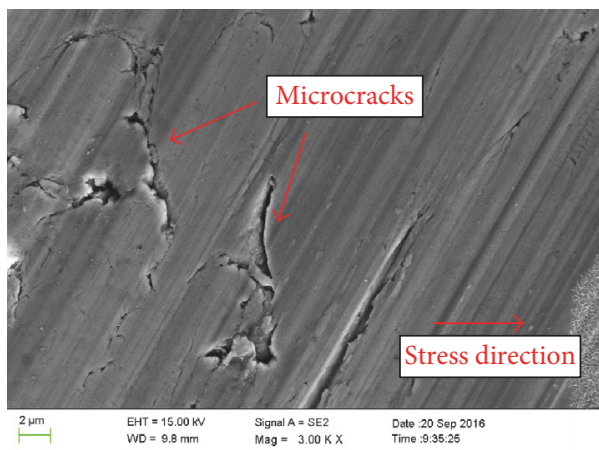

(c) Microstructure of point $\mathrm{C}$ in Figure 9

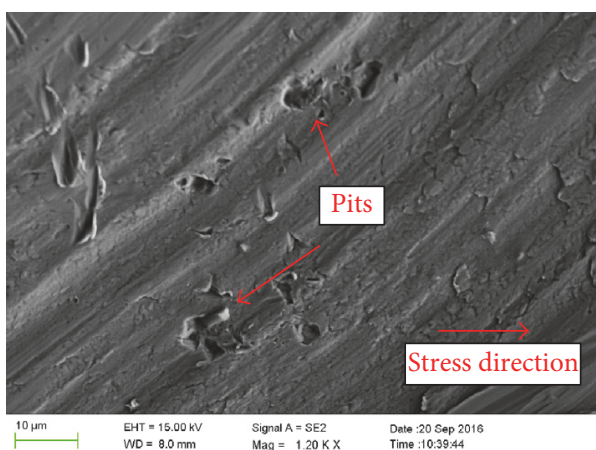

(b) Microstructure of point B in Figure 9

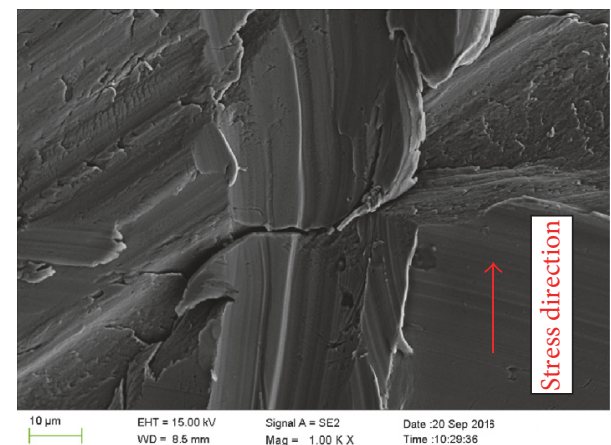

(d) Microstructure of point D in Figure 9

FIgURE 10: Microstructure of KMN-I bending fatigue specimen.

specimen increased, and the fatigue crack was more likely to occur, so that the peak of the nonlinearity parameter appeared earlier.

4.4. Microstructure Observation. In order to research the relationship between the nonlinearity parameter and the internal damage of the specimen, the microstructure of KMN-I bending specimens' cross section in the dangerous location was observed. The maximum stress is located on the surface of the middle of the specimen. So this location is the dangerous location and fatigue crack usually initiated here. After the experiment, the specimen was cut in the middle location and the cross section of the specimen was observed. Because the maximum stress is located on the surface of the specimen, we mainly observe the region near the surface in the cross section of the specimen. The cracks are mostly located on the surface and subsurface of the specimen. The results showed that the microstructure of the specimens gradually deteriorated as the fatigue cycles increased, as shown in Figure 10. The matrix of the original specimen was relatively flat and there are no obvious defects in Figure 10(a), and the nonlinearity parameter was very low in Figure 9 (point A). It was found that some small defects such as holes and pits appeared in the material matrix with the increase of the fatigue cycles in Figure 10(b), and the nonlinearity parameter increased correspondingly in Figure 9 (point B). Then the microcracks initiated from the matrix, as shown in Figure 10(c), and the nonlinearity parameter increased significantly to the peak value in Figure 9 (point C). As the fatigue cycles further increased, the cracks propagated to macrocracks, as shown in Figure 10(d). The cracks increased the attenuation coefficient of the material, so the nonlinearity parameter began to decrease in Figure 9 (point D). When the sinusoidal ultrasonic wave was transmitted into the solid medium, there will be a nonlinear interaction between the ultrasonic wave and the solid medium, resulting in the generation of high frequency harmonics. The generation of these high frequency harmonics is closely related to the microstructure of the solid medium, which is usually caused by the internal defects of the material, such as dislocations, microholes, and cracks $[3,4,10]$. In this experiment, the microstructure of the specimens gradually deteriorated with the increase of fatigue cycles, and the nonlinearity parameter and the amplitude of the second harmonic correspondingly increased. Therefore, we can conclude that these deteriorated microstructures, such as microholes and pits, are the source of the second harmonic. It is proved that there is a certain correspondence between the nonlinearity parameter and the internal damage of the material, and the nonlinearity parameter can be used to reflect the damage and fatigue life of the material.

Similarly, the microstructure of KMN-I tensile-bending specimens' cross section in the dangerous location was observed and the same law was found. The microstructure of the specimen gradually deteriorated with the increase of the fatigue cycles, as shown in Figure 11. In the early stage 


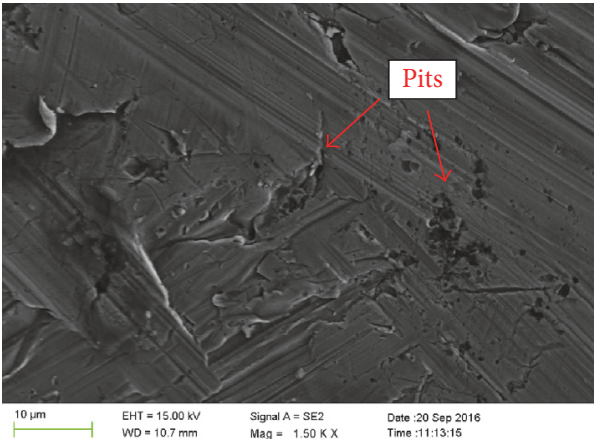

(a) Microstructure of point $\mathrm{E}$ in Figure 9

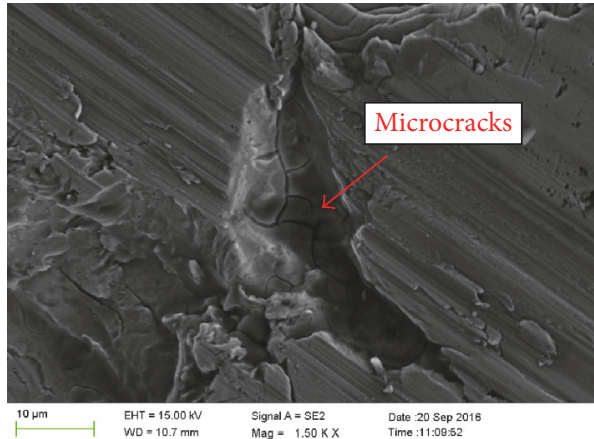

(b) Microstructure of point F in Figure 9

FIGURE 11: Microstructure of KMN-I tensile-bending fatigue specimen.

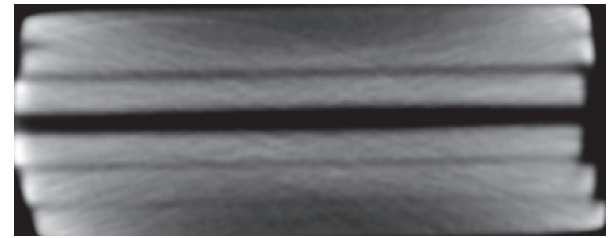

FIgURE 12: Tomography scanning of the KMN-I bending fatigue specimen.

of the fatigue life, some holes and pits can be observed in Figure 11(a). These defects evolved to microcracks under the fatigue load and the nonlinearity parameter increased correspondingly. So it is proved that the nonlinearity parameter can be used to characterize the fatigue life of the material.

Industrial CT can display the internal structure, composition, material, and defect status of the object clearly, accurately, and intuitively in the form of two-dimensional tomographic image or three-dimensional image. It is a commonly used nondestructive testing and evaluation technique. Tomography scanning was carried out to the specimens with different fatigue damage using industrial CT (resolution $0.1667 \mathrm{~mm}$ ). No obvious defects were found, as shown in Figure 12. But in SEM micrographs we can observe many small defects in the specimen and the nonlinear parameter increased correspondingly. So we can conclude that these small defects which cannot be detected by the traditional nondestructive testing can result in the changes of the nonlinearity parameter, which proves that the nonlinearity parameter is more sensitive to small defects and the nonlinear ultrasonic testing can be used for the nondestructive testing in the early stage of the fatigue life.

\section{Discussion}

5.1. $\beta$-a Curve. With the increase of the fatigue cycles, the microstructure defects evolved to cracks and finally led to the failure of the material. So the increase of the fatigue cycles was also the initiation and propagation process of the fatigue crack. Combined with the microstructure analysis of the bending fatigue specimen in the dangerous location, the number and length of the fatigue cracks in ten standard fields of view were statistically counted, as shown in Table 4, where the equivalent crack length was the sum of the cracks' lengths in the field of view. Since the received nonlinear ultrasonic Lamb signal was the sum of the second harmonics generated by all the defects in the sampling region, it was more reasonable to use the equivalent crack length compared to the single crack length in the research. From Figure 13, it can be seen that the number of cracks and equivalent crack size increased with the increase of fatigue cycles. Especially in the early stage of fatigue life, the nonlinearity parameter is positively correlated with the equivalent crack size. Therefore, we can think that the changes of the number and size of microcracks result in the change of nonlinearity parameter.

In order to analyse the relationship between the microcracks of the specimen and the measured nonlinearity parameter, we establish the relationship curve between the equivalent crack length and the nonlinearity parameter ( $\beta$ - $a$ curve), as shown in Figure 14. Due to the decrease of the nonlinearity parameter caused by the signal attenuation of the material in the late stage of the fatigue life, we focused on the correspondence in the early stage of the fatigue life. It can be found that the nonlinearity parameter of the material increased with the increase of the crack length, which indicated that the nonlinearity parameter can reflect the propagation of the internal fatigue crack.

In addition, the tensile-bending fatigue test showed a similar law to the bending fatigue test. The microstructure of the specimens gradually deteriorated with the increase of fatigue cycles and the nonlinearity parameter and the amplitude of the second harmonic correspondingly increased in the early stage of the fatigue life.

5.2. Changes of Material Strength and Hardness. According to the nonlinearity parameter-fatigue cycles curve ( $\beta-N$ curve) obtained above, we can use the nonlinear ultrasonic Lamb wave measurement to determine the fatigue damage and fatigue life of the material. Due to the characteristics of KMN-I steel, the $\beta-N$ curve showed a trend to increase first and then decrease. Therefore, the nonlinearity parameter obtained in the actual measurement may correspond to two fatigue cycles, and the difference between the two fatigue 
TABLE 4: Statistics of the cracks in the specimens.

\begin{tabular}{lccccccc}
\hline Fatigue cycles & $5 \times 10^{4}$ & $10^{5}$ & $5 \times 10^{5}$ & $7 \times 10^{5}$ & $10^{6}$ & $5 \times 10^{6}$ & $10^{7}$ \\
\hline Number of cracks & 0 & 0 & 1 & 3 & 4 & 6 & 12 \\
Length of cracks & 0 & 0 & 8 & $12,6,7$ & $18,8,4,26$ & $30,12,25,10,14,16$ & $9,7,31,13,8,11,25,58,5,11,12$ \\
Equivalent crack length & 0 & 0 & 8 & 25 & 56 & 107 & 181 \\
\hline
\end{tabular}

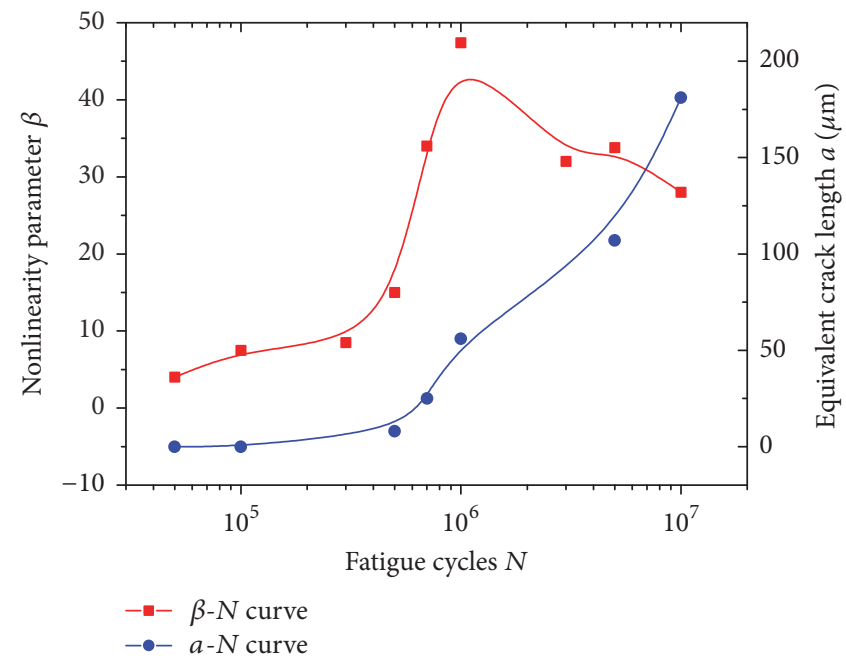

FIGURE 13: Relationship between equivalent crack length and fatigue cycles.

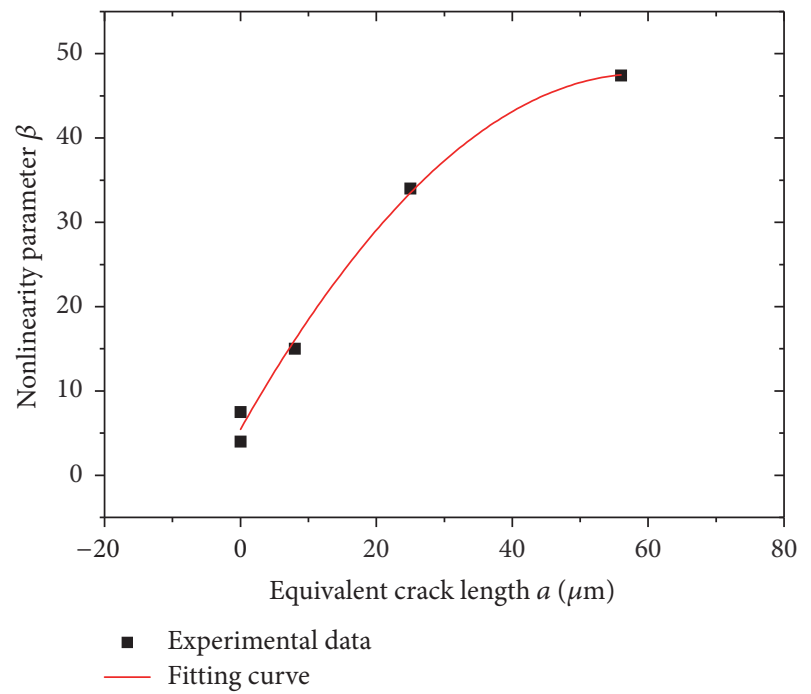

FIGURE 14: Relationship between equivalent crack length and nonlinearity parameter.

cycles may be very large. One solution is to continue loading for a period of time after nonlinear ultrasonic testing and then carry out nonlinear ultrasonic testing again. We compare the differences between the two results to determine whether the material is currently in the rise or fall stage of the $\beta$ $N$ curve, so that we can accurately locate the fatigue cycle of the material. In addition, we can add a characterization parameter that combines the nonlinearity parameter to locate the fatigue cycles. The tensile strength and hardness of the two groups of specimens with different fatigue cycles were measured to study whether there was a certain relationship between the two parameters and the fatigue cycles.

First, the tensile strength of the specimens was measured using ABI test system. There was no obvious trend between the tensile strength and the fatigue cycle, as shown in Figure 15. So it was difficult to judge the fatigue degree of the material by the tensile strength.

Secondly, the hardness of the specimens was measured. It was found that the hardness of the material increased with the increase of the fatigue cycle, which indicated that KMN-I showed cycle hardening behavior, as shown in Figure 16. The hardness of the material will increase $5 \%-10 \%$ in the late stage of the fatigue life ( $>10^{6}$ cycles). So we can use the change of the hardness to characterize the fatigue degree of the material. When the hardness increased by more than $5 \%$, the material was in the late stage of the fatigue life, that is, the fall stage of the $\beta-N$ curve. On the contrary, we thought that the material was in the early stage of the fatigue life, that is, the rise stage of the $\beta-N$ curve.

Taking the KMN-I bending fatigue specimen as an example, combined with the $\beta-N$ curve and the hardness curve, we can establish a double criterion method for estimating the fatigue life of the material using the nonlinearity parameter, as shown in Figure 17. Firstly, the nonlinear ultrasonic testing was carried out on the material after service, and the nonlinearity parameter was obtained, which corresponded to two possible fatigue cycles in the $\beta-N$ curve. Then hardness of the material was measured, and the fatigue life can be accurately determined according to the change of the hardness. If the hardness increased by more than 5\% (blue dots), the fatigue 


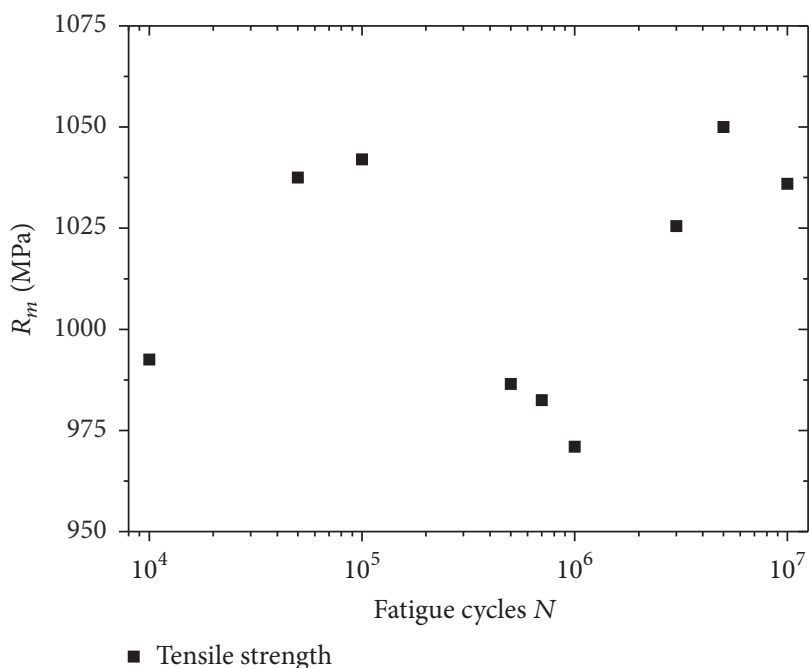

(a) Bending fatigue test

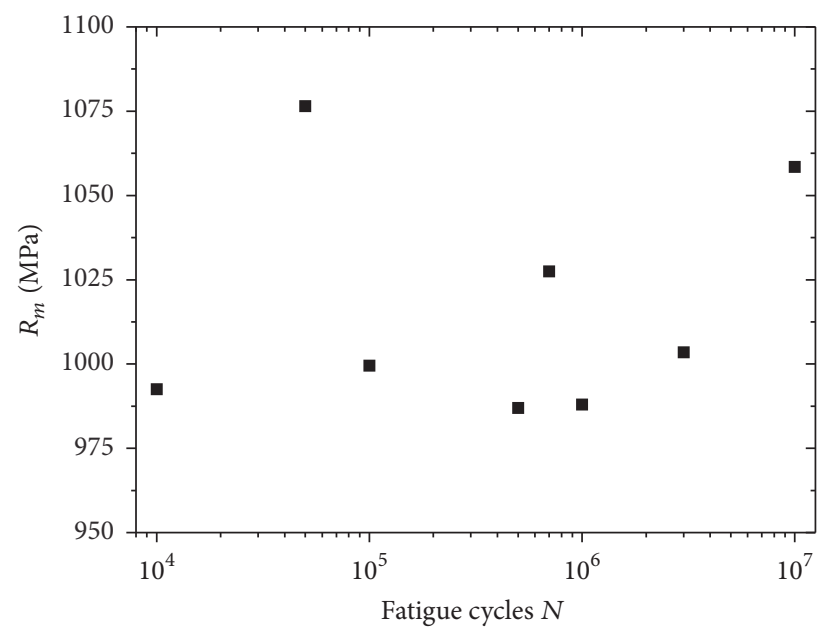

- Tensile strength

(b) Tensile-bending fatigue test

FIGURE 15: Tensile strength of two groups of specimens.

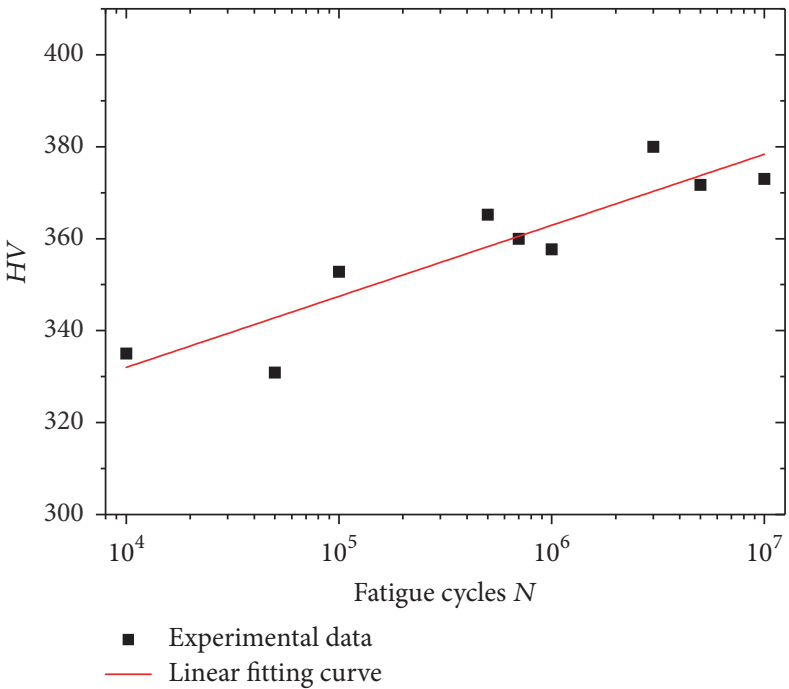

(a) Bending fatigue test

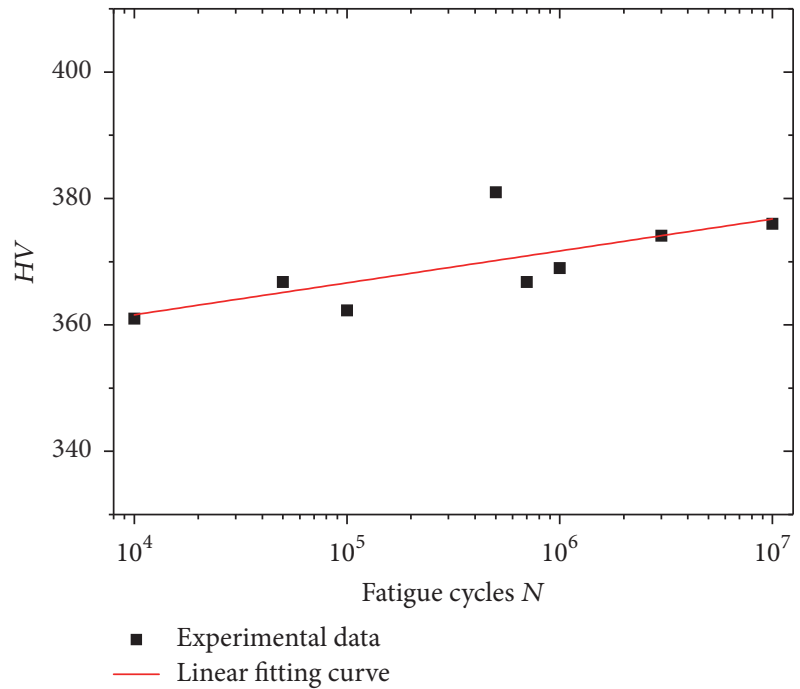

(b) Tensile-bending fatigue test

FIGURE 16: Hardness of two groups of specimens.

cycles should be in the fall stage of the $\beta-N$ curve, while if the hardness increased by less than $5 \%$ (black dots), the fatigue cycles should be in the rise stage of the $\beta-N$ curve.

\section{Conclusions}

(1) The nonlinear ultrasonic testing was carried out on two groups of specimens with different fatigue loads (bending fatigue test and tensile-bending fatigue test), and the $\beta-N$ curve of the compressor blade steel KMN-I was obtained. It was found that the nonlinearity parameter of the material increased first and then decreased with the increase of the fatigue cycles.
(2) The microstructures were observed by scanning electron microscopy (SEM). The results showed that, with the increase of the fatigue cycles, the microstructure of the material gradually deteriorated and then the microcracks initiated from the matrix. It was proved that there was a certain correspondence between the nonlinearity parameter and the internal fatigue damage of the material, and the nonlinearity parameter can be used to characterize the fatigue damage and fatigue life of the material.

(3) The relationship between the propagation of the internal cracks and the nonlinearity parameter was analysed. Based on the $\beta-N$ curve and hardness test of the material, the method of evaluating the fatigue cycles of the material was proposed. 


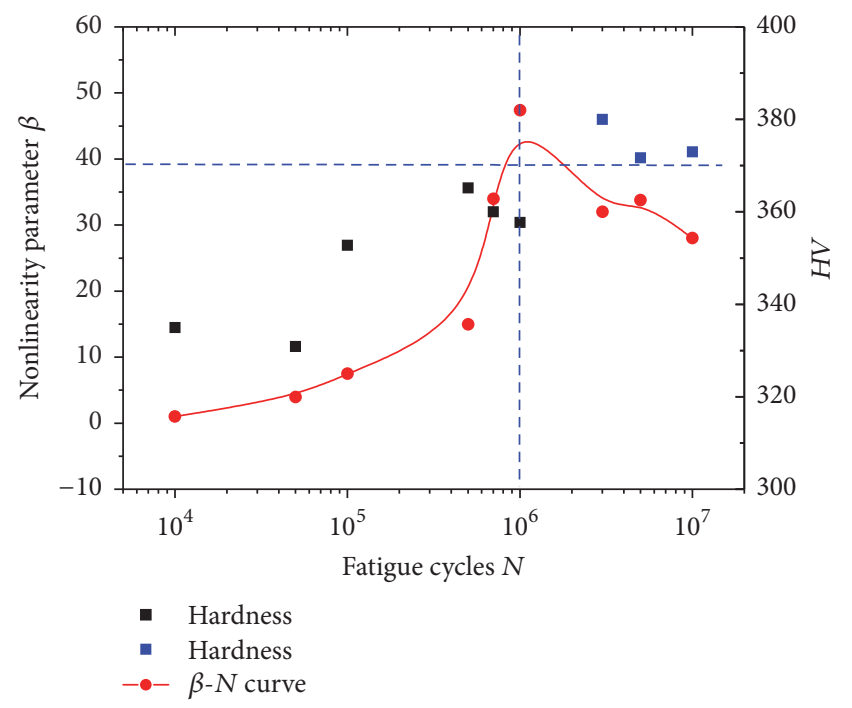

FIGURE 17: Relationship between hardness and nonlinearity parameter of KMN-I bending fatigue specimen.

\section{Conflicts of Interest}

The authors declare that they have no conflicts of interest.

\section{Acknowledgments}

The authors gratefully acknowledge the financial support of the National Key Basic Research and Development Program (973 Program), through Grant no. 2011CB013401.

\section{References}

[1] J. H. Cantrell, "Substructural organization, dislocation plasticity and harmonic generation in cyclically stressed wavy slip metals," Proceedings of the Royal Society A Mathematical, Physical and Engineering Sciences, vol. 460, no. 2043, pp. 757-780, 2004.

[2] T. Ohtani, "Acoustic damping characterization and microstructure evolution during high-temperature creep of an austenitic stainless steel," Metallurgical and Materials Transactions A: Physical Metallurgy and Materials Science, vol. 36, no. 11, pp. 2967-2977, 2005.

[3] J. Herrmann, J.-Y. Kim, L. J. Jacobs, J. Qu, J. W. Littles, and M. F. Savage, "Assessment of material damage in a nickel-base superalloy using nonlinear Rayleigh surface waves," Journal of Applied Physics, vol. 99, no. 12, Article ID 124913, 2006.

[4] J.-Y. Kim, L. J. Jacobs, J. Qu, and J. W. Littles, "Experimental characterization of fatigue damage in a nickel-base superalloy using nonlinear ultrasonic waves," The Journal of the Acoustical Society of America, vol. 120, no. 3, pp. 1266-1273, 2006.

[5] B. S. Yan, B. Wu, and X. C. Zeng, "FEM simulation and experimental study of fatigue damage measurement in magnesium using nonlinear ultrasonic," Shengxue Xuebao/acta Acustica, vol. $5,2010$.

[6] Z. Zhou and S. Liu, "Nonlinear ultrasonic techniques used in nondestructive testing: A review," Jixie Gongcheng Xuebao/ Journal of Mechanical Engineering, vol. 47, no. 8, pp. 2-11, 2011.
[7] K.-Y. Jhang and K.-C. Kim, "Evaluation of material degradation using nonlinear acoustic effect," Ultrasonics, vol. 37, no. 1, pp. 39-44, 1999.

[8] K. Kawashima, T. Ito, and Y. Nagata, "Detection and imaging of nonmetallic inclusions in continuously cast steel plates by higher harmonics," Japanese Journal of Applied Physics, vol. 49, no. 7, Article ID 07HC11, 2010.

[9] M. Cremer, M. Zimmermann, and H. Christ, In-situ characterization of the damage evolution of welded aluminum alloy joints during very high cycle fatigue (VHCF) with nonlinear ultrasonic technique, Supplemental Proceedings: Materials Properties, Characterization, and Modeling, vol. 2, John Wiley \& Sons, Inc., 2012.

[10] W. Li, H. Cui, W. Wen, X. Su, and C. C. Engler-Pinto, "In situ nonlinear ultrasonic for very high cycle fatigue damage characterization of a cast aluminum alloy," Materials Science and Engineering: A Structural Materials: Properties, Microstructure and Processing, vol. 645, pp. 248-254, 2015.

[11] I. Y. Solodov, "Nonlinear NDE using contact acoustic nonlinearity (CAN)," in Proceedings of the 1994 IEEE Ultrasonics Symposium. Part 1 (of 3), pp. 1279-1283, November 1994.

[12] I. Y. Solodov, N. Krohn, and G. Busse, "CAN: An example of nonclassical acoustic nonlinearity in solids," Ultrasonics, vol. 40, no. 1-8, pp. 621-625, 2002.

[13] Q. Yao and J. Yao, "Vibration fatigue in engineering structures," Yingyong Lixue Xuebao/Chinese Journal of Applied Mechanics, vol. 23, no. 1, pp. 12-15, 2006.

[14] R. Rajasekaran and D. Nowell, "Fretting fatigue in dovetail blade roots: Experiment and analysis," Tribology International, vol. 39, no. 10, pp. 1277-1285, 2006.

[15] W. G. Liu and H. L. He, "Resonance fatigue testing and analysis of one-way double-stiffened plate," Journal of Experimental Mechanics, vol. 27, no. 3, pp. 361-367, 2012.

[16] K. H. Matlack, J.-Y. Kim, L. J. Jacobs, and J. Qu, "Review of Second Harmonic Generation Measurement Techniques for Material State Determination in Metals," Journal of Nondestructive Evaluation, vol. 34, no. 1, 2015.

[17] A. J. Croxford, P. D. Wilcox, B. W. Drinkwater, and P. B. Nagy, "The use of non-collinear mixing for nonlinear ultrasonic detection of plasticity and fatigue," The Journal of the Acoustical Society of America, vol. 126, no. 5, pp. EL117-EL122, 2009.

[18] J. H. Cantrell and W. T. Yost, "Nonlinear ultrasonic characterization of fatigue microstructures," International Journal of Fatigue, vol. 23, no. 1, pp. S487-S490, 2001.

[19] Y. X. Xiang, Evaluation of early damage in high-temperature components based on nonlinear ultrasonic guided waves [Doctoral, thesis], East China University of Science and Technology, 2011.

[20] M. Deng, "Analysis of second-harmonic generation of Lamb modes using a modal analysis approach," Journal of Applied Physics, vol. 94, no. 6, pp. 4152-4159, 2003.

[21] A. Hikata, B. B. Chick, and C. Elbaum, "Dislocation contribution to the second harmonic generation of ultrasonic waves," Journal of Applied Physics, vol. 36, no. 1, pp. 229-236, 1965.

[22] A. Hikata, F. A. Sewell, and C. Elbaum, "Generation of ultrasonic second and third harmonics due to dislocations. II," Physical Review A: Atomic, Molecular and Optical Physics, vol. 151, no. 2, pp. 442-449, 1966. 


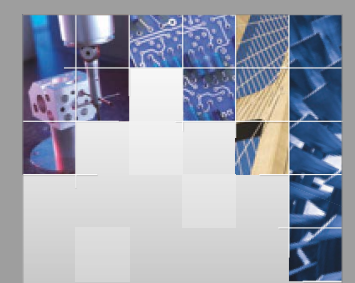

\section{Enfincering}
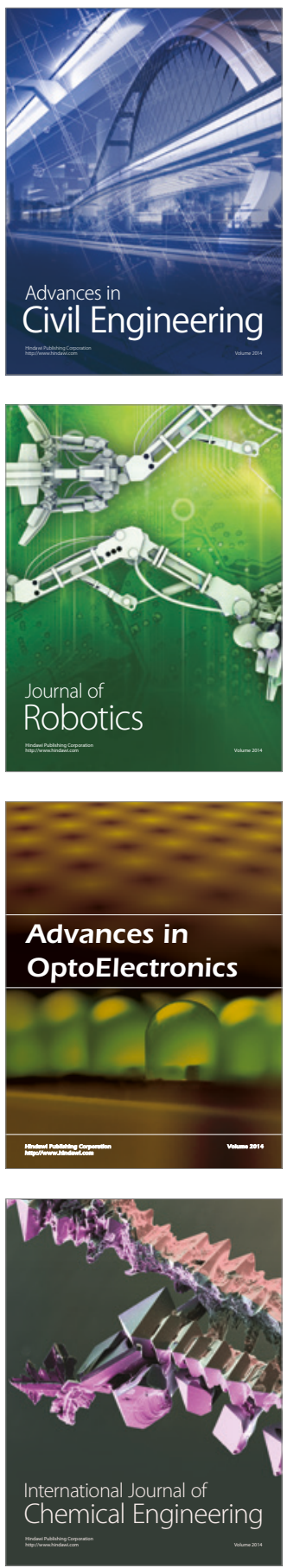

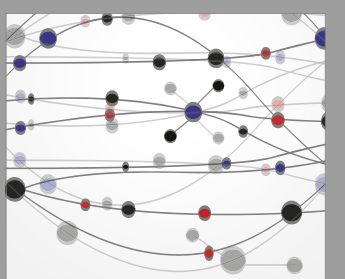

The Scientific World Journal

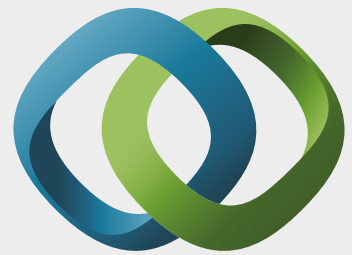

\section{Hindawi}

Submit your manuscripts at

https://www.hindawi.com
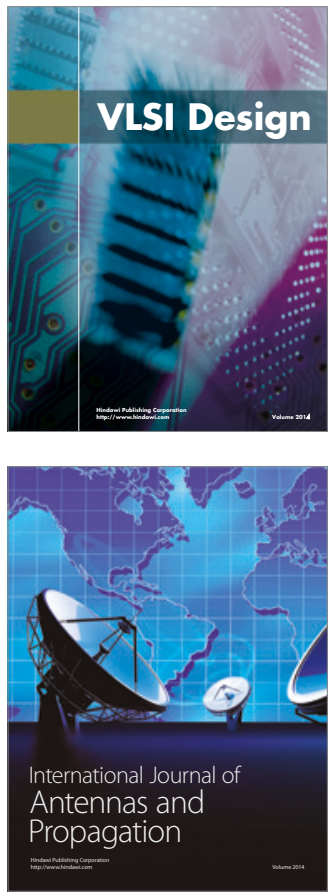

\section{Rotating}

Machinery
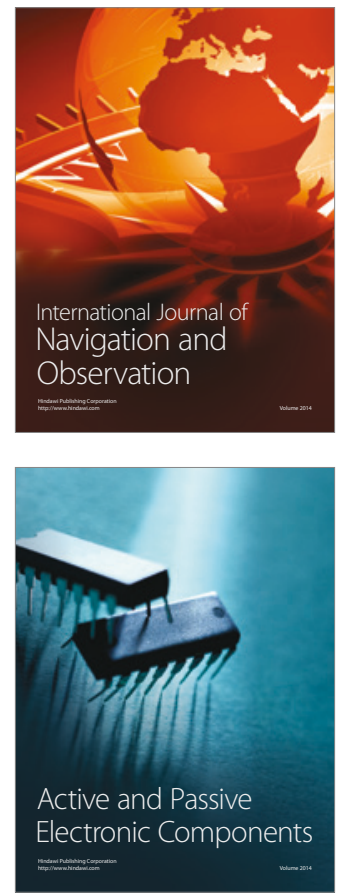
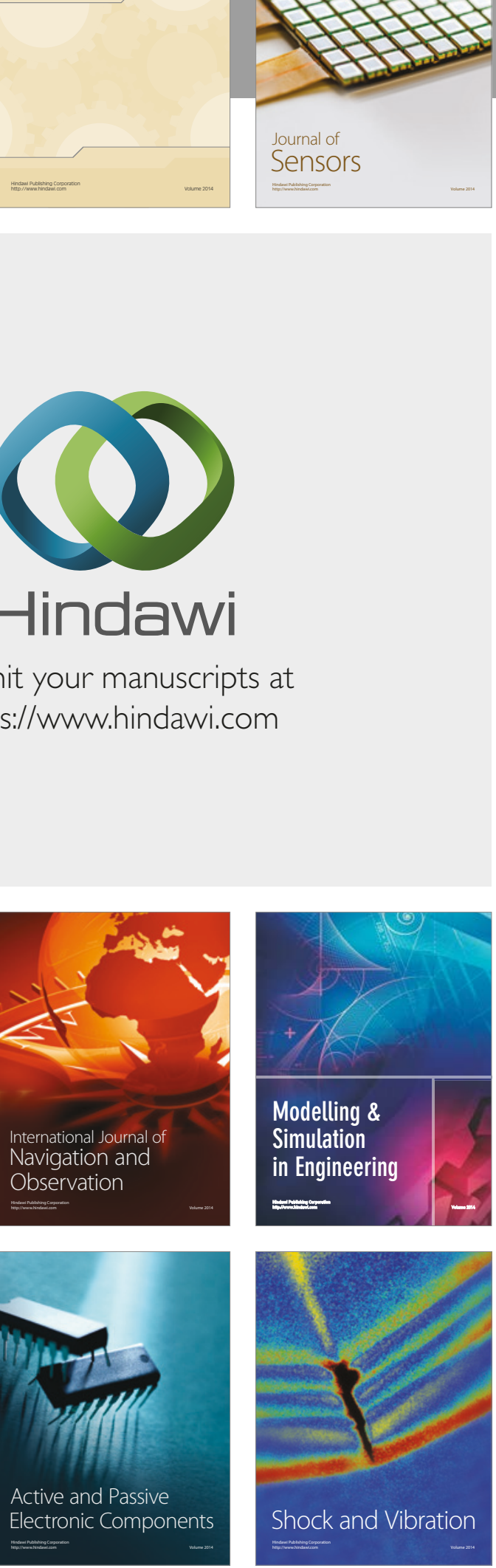
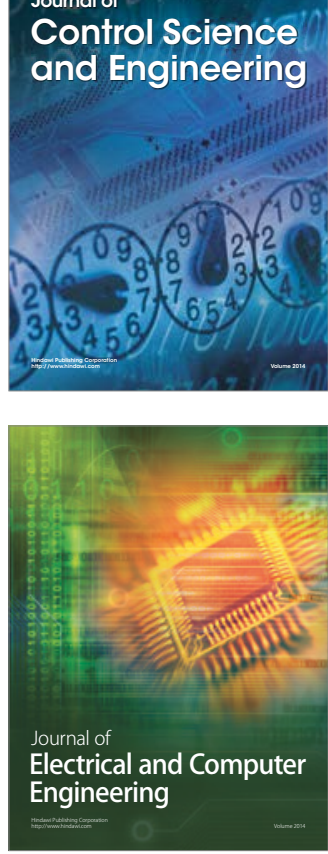

Distributed

Journal of

Control Science

and Engineering
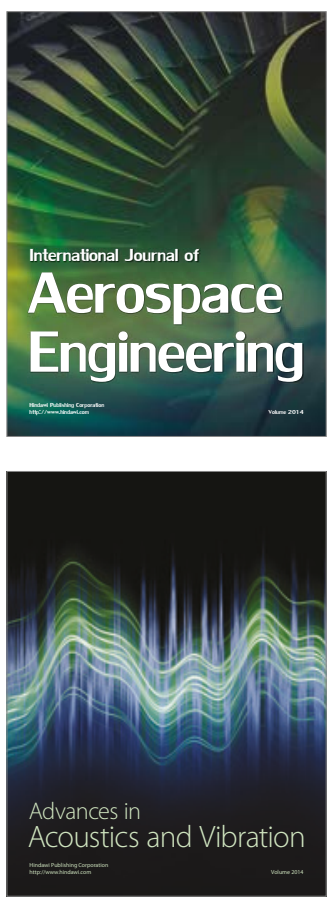

Sensor Networks 